\title{
Impacto de intervenção educativa na melhoria do Nursing Activities Score em
}

\section{Neonatologia}

\author{
Impact of an educational intervention on improving the Nursing Activities Score in Neonatology \\ Impacto de una intervención educativa en la mejora del Nursing Activities Score en Neonatología
}

Recebido: 11/11/2021 | Revisado: 19/11/2021 | Aceito: 14/12/2021 | Publicado: 22/12/2021

Eduarda Bandeira Pereira

ORCID: https://orcid.org/0000-0003-0492-588X Universidade Federal do Rio Grande do Sul, Brasil E-mail: dudabp@hotmail.com

Gisela Maria Schebella Souto de Moura

ORCID: https://orcid.org/0000-0002-4744-478X Universidade Federal do Rio Grande do Sul, Brasil E-mail: gisela.moura@ufrgs.br

Ana Cristina Pretto Báo

ORCID: https://orcid.org/0000-0002-2747-7197 Universidade Federal do Rio Grande do Sul, Brasil E-mail: anacbao@yahoo.com.br

Márcia Koja Breigeiron

ORCID: https://orcid.org/0000-0001-6546-8868 Universidade Federal do Rio Grande do Sul, Brasil E-mail: mbreigeiron@gmail.com

Elenice Lorenzi Carniel

ORCID: https://orcid.org/0000-0002-6611-8525 Hospital de Clínicas de Porto Alegre, Brasil E-mail: ecarniel@hcpa.edu.br

Ana Paula Morais de Carvalho Macedo

ORCID: https://orcid.org/0000-0002-1064-3523

Universidade do Minho, Portugal

E-mail: amacedo@ese.uminho.pt

Louíse Viecili Hoffmeister

ORCID: https://orcid.org/0000-0002-8858-5693 Universidade NOVA de Lisboa, Portugal

Comprehensive Health Research Center, Portugal Nursing Research, Innovation and Development Centre of Lisbon, Portugal E-mail: 1v.hoffmeister@ensp.unl.pt

\begin{abstract}
Resumo
Objetivo: Comparar os escores do Nursing Activities Score (NAS) obtidos nos anos de 2015 e 2016 em uma UTI Neonatal (UTIN) após intervenção educativa com enfermeiras. Método: Estudo com delineamento retrospectivo, comparativo, do tipo antes e depois. Amostra constituída por dados secundários dos escores de NAS, de janeiro de 2015 a dezembro de 2016. Análise dos dados por meio da estatística descritiva e do teste t-Student $(\mathrm{P}<0,05)$. Resultados: Escores anuais médios revelaram valores de 70,5 (2015) e 78,8 (2016), evidenciando que as horas de assistência de enfermagem demandas pelos pacientes passaram de 16,98 para 19,06 horas. No período pós-intervenção, o valor mensal médio de NAS foi 84,6 (2016), enquanto no mesmo período do ano anterior havia sido 69,3. A conversão desses valores representa 16,63 horas (2015) e 20,03 horas (2016). Conclusão: Salienta-se o impacto positivo advindo da intervenção educativa, ratificando a necessidade da educação permanente para os profissionais de saúde.

Palavras-chave: Carga de trabalho; Neonatologia; Educação continuada; Enfermagem; Melhoria de qualidade.

Abstract

Aim: To compare the Nursing Activities Score (NAS) scores obtained in 2015 and 2016 in a Neonatal ICU (NICU) after an educational intervention with nurses. Method: Retrospective, comparative, before-and-after study. Sample consisted of secondary data from NAS scores, from January 2015 to December 2016. Data analysis through descriptive statistics and t-Student test $(\mathrm{P}<0.05)$. Results: Mean annual scores revealed values of 70.5 (2015) and 78.8 (2016), evidencing that the hours of nursing care demanded by patients increased from 16.98 to 19.06 hours. In the postintervention period, the average monthly NAS value was 84.6 (2016), while in the same period of the previous year it had been 69.3. The conversion of these values represents 16.63 hours (2015) and 20.03 hours (2016). Conclusion: The positive impact arising from the educational intervention is highlighted, ratifying the need for permanent education for health professionals.
\end{abstract}

Keywords: Workload; Neonatology; Education continuing; Nursing; Quality improvement. 


\begin{abstract}
Resumen
Objetivo: Comparar las puntuaciones del Nursing Activities Score (NAS) obtenidas en 2015 y 2016 en una UCI neonatal (UCIN) tras una intervención educativa con enfermeras. Método: Estudio retrospectivo, comparativo, antes y después. La muestra consistió en datos secundarios de las puntuaciones del NAS, desde enero de 2015 hasta diciembre de 2016. Análisis de datos mediante estadística descriptiva y prueba t-Student $(\mathrm{P}<0,05)$. Resultados: Las puntuaciones medias anuales revelaron valores de 70,5 (2015) y 78,8 (2016), evidenciando que las horas de cuidados de enfermería demandadas por los pacientes aumentaron de 16,98 a 19,06 horas. En el periodo posterior a la intervención, el valor medio mensual del NAS fue de 84,6 (2016), mientras que en el mismo periodo del año anterior había sido de 69,3. La conversión de estos valores representa 16,63 horas (2015) y 20,03 horas (2016). Conclusión: Se salienta el impacto positivo adviniendo de la intervención educativa, ratificando la necesidad de la educación permanente para los profesionales de la salud.
\end{abstract}

Palabras clave: Carga de trabajo; Neonatología; Educación continua; Enfermería; Mejoramiento de la calidad.

\title{
1. Introdução
}

$\mathrm{Na}$ assistência de enfermagem a pacientes críticos, a carga de trabalho oriunda das demandas assistenciais e o dimensionamento de pessoal de enfermagem são temáticas importantes que permeiam a rotina diária dos enfermeiros (MendesRodrigues et al., 2017). Neste contexto, a utilização de instrumentos capazes de quantificar a demanda de cuidados é fundamental para adequação da equipe de enfermagem, considerando a possibilidade do planejamento da assistência o mais próximo da realidade (Oliveira et al., 2019)

O Conselho Federal de Enfermagem (COFEN), por meio da Resolução 0543/2017, atualiza e estabelece os parâmetros para o dimensionamento do quadro de profissionais de enfermagem nos serviços/locais em que são realizadas atividades de enfermagem. O quantitativo de horas de enfermagem, por paciente, nas 24 horas, preconizadas incluem: 4 horas de enfermagem no cuidado mínimo; 6 horas de enfermagem no cuidado intermediário; 10 horas de enfermagem no cuidado de alta dependência, 10 horas de enfermagem no cuidado semi-intensivo e 18 horas de enfermagem no cuidado intensivo. Recém-nascidos (RN) e menores de seis anos de idade são classificados, no mínimo, como cuidado intermediário, independente da presença do acompanhante (COFEN, 2017).

No Brasil, há cerca de uma década, o Ministério da Saúde determinou a obrigatoriedade da classificação dos pacientes em Unidade de Tratamento Intensivo (UTI), normatização ainda válida para os dias atuais. (Brasil, 2010). Diante da determinação ministerial, os enfermeiros brasileiros viram-se compelidos a optarem por uma das escalas validadas no país e, desta forma, o Nursing Activities Score (NAS) tornou-se um instrumento amplamente utilizado no contexto do intensivismo nacionalmente.

O NAS é um instrumento para avaliação da carga de trabalho de enfermagem (CTE) em UTI, composto por 23 itens, cujos pesos variam de um mínimo de 1,2 a um máximo de 32 e possui em sua estrutura sete domínios, sendo eles: atividades básicas, suporte ventilatório, cardiovascular, renal, neurológico, metabólico e intervenções específicas. Ao multiplicar o total de pontos resultantes da avaliação pela constante de 14,4 obtém-se o quantitativo de horas de enfermagem que o paciente necessita (Queijo \& Padilha, 2009).

Conquistando um reconhecimento mundial (Bruyneel et al., 2019), o instrumento tem sido incorporado por hospitais em diferentes países, com utilização na área de gestão de pessoas, assistencial e financeira, contribuindo para fundamentar a delicada discussão da resultante da tríade entre qualidade assistencial X composição da equipe de enfermagem X custos (Santos et al., 2021; Bruyneel et al., 2019; Castro et al., 2018). Além disso, a CTE influencia diretamente a saúde do trabalhador, pois, quando há sobrecarga laboral, o profissional pode ser levado à exaustão emocional e adoecimento, desenvolvendo Síndrome de Burnout, quadro relativamente recorrente em enfermeiros de unidades intensivas (Aragão et al., 2021; Biff et al., 2020; Santos et al., 2021). Especificamente em unidade neonatal, a literatura descreve a sobrecarga de trabalho como um risco ocupacional que pode gerar dano físico, mas identifica também a presença de estresse ocupacional, exaustão emocional, Burnout, desmotivação e dificuldades ne relacionamento, dentre outras manifestações (Silva et al., 2021).

Na UTI Neonatal (UTIN) o avanço do conhecimento científico e a utilização de novas tecnologias têm contribuído para 
o aumento da sobrevida dos RN, especialmente dos prematuros extremos e de extremo baixo peso, provocando impactantes mudanças no cenário da assistência neonatal (Bochembuzio, 2007; Branco et al., 2017; Tomazoni et al., 2017). Se por um lado o avanço na assistência trouxe novas possibilidades terapêuticas e de vida aos pequenos e frágeis RN, por outro, esse mesmo avanço incorporou maiores riscos assistenciais com a utilização de potentes medicamentos e equipamentos terapêuticos complexos que exigem maior atenção e conhecimento dos profissionais que estão na linha de frente do atendimento hospitalar.

Os riscos assistenciais e/ou a ocorrência de eventos adversos têm sido preocupação de estudos acerca da qualidade do atendimento hospitalar e da segurança dos pacientes. Em publicação recente, pesquisadores analisaram os tipos de incidentes notificados em uma unidade de cuidados neonatais, encontrando que $83,3 \%$ dos incidentes ocorridos estavam relacionados a cuidados de enfermagem, como administração errada de medicamento, perda acidental de tubo traqueal, obstrução de cateter por pausa prolongada de infusão, lesão de pele, entre outros (Hoffmeister et al., 2019).

A vinculação de temas inseridos na segurança do paciente à carga de trabalho da equipe de enfermagem e a adequação das equipes frente às demandas assistenciais tem aumentado o interesse e a complexidade dos estudos no Brasil. A análise de indicadores assistenciais e carga de trabalho mostra que maiores proporções no número de pacientes designados aos profissionais de enfermagem estão associadas a um aumento nos dias de permanência hospitalar e aumento das taxas de infecção urinária dos pacientes (Magalhães et al., 2017).

No âmbito da assistência neonatal, equipes de enfermagem com quantitativo insuficiente de profissionais, insumos materiais inadequados e más condições do ambiente de trabalho são fatores de risco que comprometem a qualidade do atendimento de enfermagem e a segurança dos pacientes reconhecidos pela equipe de enfermagem (Tomazoni et al., 2017). A estrutura física inadequada, aliada a protocolos e rotinas institucionais deficientes foram identificados como fatores de risco para ocorrência de incidentes no processo de medicação em neonatologia (Guzzo et al., 2018).

Em virtude da relevância da contribuição do NAS para o adequado dimensionamento da equipe de enfermagem para a prestação de cuidados com qualidade e segurança em unidades intensivas neonatais destaca-se a necessidade da aplicação correta dessa escala para que a mesma represente a real complexidade assistencial.

Mensurações subestimadas da CTE impactam na qualidade e segurança dos cuidados para os pacientes e para os profissionais, assim como mensurações superestimadas podem ocasionar alocações inadequadas de profissionais e aumento de custos diretamente relacionados com assistência (Santos et al., 2021; Bruynnel et al., 2019; Castro et al., 2018). Diante dessa ótica, os profissionais envolvidos na utilização dessa escala devem estar devidamente habilitados e possuir conhecimentos sobre os itens que compõem o instrumento para que possam ajustar o número de profissionais para atender às demandas requeridas pelos pacientes, medidas por meio da aplicação do NAS (Oliveira et al., 2019).

$\mathrm{Na}$ unidade estudada percebeu-se que as avaliações da CTE realizadas pelas enfermeiras pareciam não retratar a realidade da demanda de cuidados dos RN, optando-se pela realização de estratégia educativa com os enfermeiros, para aprimorar suas habilidades na classificação dos pacientes. Em estudo realizado no sul do Brasil, os autores referem que a realização de atividades educativas em saúde favorece as modificações nas práticas profissionais, assim como melhorias na organização do trabalho nas instituições (Kleba et al., 2017). Nesse mesmo sentido, pesquisa recente em uma unidade neonatal apontou que o trabalho da equipe de enfermagem demanda um processo contínuo de atualização, pautado em um plano de estratégias pedagógicas que levem em consideração a dinâmica do cotidiano do processo de trabalho (Silva et al., 2018).

Assim, instigadas pelos questionamentos advindos da prática profissional da aparente inadequação dos escores de NAS e a necessidade de buscar evidências para fundamentar a composição da equipe de enfermagem de uma UTIN, as autoras implementaram ação educativa focada no NAS. Uma vez concluído o processo educativo, os escores de NAS foram analisados por meio de abordagem comparativa. Neste sentido, o presente estudo teve como objetivo comparar os escores do NAS obtidos antes e após uma intervenção educativa com enfermeiras de uma UTIN. 


\section{Metodologia}

Trata-se de um estudo com abordagem quantitativa, de delineamento retrospectivo e comparativo, com realização de intervenção e avaliação antes e depois. No desenvolvimento, foram respeitados os preceitos da guideline SQUIRE 2.0 (estrutura para relatar conhecimentos sobre melhoria da qualidade em saúde).

Este estudo foi desenvolvido em um hospital universitário da Região Sul do Brasil, que atende os pacientes pelo Sistema Único de Saúde (SUS), referência no atendimento de excelência e alta complexidade em amplo rol de especialidades, em especial, para o presente estudo, na área da gestação de alto risco. A instituição conta com UTIN, a qual atende RN, prematuros (bebês que nascem antes de 37 semanas de idade gestacional) e a termo (bebês que nascem entre a $38^{\mathrm{a}}$ e a $41^{\mathrm{a}}$ semanas de idade de gestação), portadores das mais diversas patologias e que demandem cuidados intensivos e intermediários, nascidos na instituição ou procedentes de outros hospitais. Trata-se de uma área de atendimento neonatal composta por 50 leitos, sendo 20 leitos da UTIN nível III, que atende pacientes de alta complexidade, 20 leitos da Unidade de Cuidado Intermediário Neonatal Convencional (UCINCo) e 10 leitos da Unidade de Cuidado Intermediários Canguru (UCINCa). A equipe de enfermagem da unidade neonatal é composta por 42 enfermeiros e 95 técnicos de enfermagem, mantendo-se inalterada durante os anos de 2015 e 2016. Para os leitos de UTI, a equipe conta com 25 enfermeiros e 58 técnicos de enfermagem.

Cabe esclarecer que, periodicamente, os RN internados nos leitos de intensivismo são avaliados pelos enfermeiros nos domínios integrantes do NAS, na frequência de uma avaliação a cada 24 horas, havendo uma definição prévia dos responsáveis pela atividade. Essa avaliação é feita realizando um exame físico no RN e inspeção nos equipamentos em uso para manutenção de suas condições vitais e terapêuticas, além de uma busca retrospectiva nas evoluções e prescrições do recém-nascido internado nas 24 horas antecedentes à avaliação. Posteriormente, registram-se os valores de NAS em um formulário eletrônico online. O instrumento é aplicado somente pelos enfermeiros e em pacientes internados na UTI neonatal, pois tão logo sejam transferidos para um leito de cuidados intermediários, é registrada a alta do NAS no sistema institucional.

A intervenção educativa denominada "Preenchimento do NAS no sistema" foi realizada na unidade de neonatologia, no mês de maio de 2016, em diferentes turnos de trabalho, para abranger as diversas equipes de enfermeiros do setor, com duração de aproximadamente duas horas cada. A capacitação utilizou-se da estratégia de Grupos Focados para atualizar e homogeneizar as avaliações dos enfermeiros. Na prática, isto significa que o tema foi abordado de forma teórica e discutido no grupo com os participantes, durante o turno de trabalho dos enfermeiros, permitindo o esclarecimento de dúvidas e resultando em consenso grupal. Na sequência deste processo, ocorria a demonstração do preenchimento online do instrumento no sistema do hospital (Intranet) para os enfermeiros presentes. A atividade foi de cunho setorial e baseada no tutorial desenvolvido por Bochembuzio (2007) sobre o uso correto do instrumento. Todos os enfermeiros foram convidados a participar da intervenção educativa. A capacitação abrangeu aproximadamente $72 \%$ da equipe de enfermeiros (30 profissionais) e não acarretou custos adicionais para o setor.

A coleta de dados ocorreu de maio a julho de 2017 por meio de consulta eletrônica no sistema informatizado da instituição, onde está localizado o banco de dados do NAS da UTIN. Os dados incluíram todos os valores diários do NAS referentes à avaliação dos RN internados nos leitos intensivos entre janeiro de 2015 e dezembro de 2016. A coleta foi realizada por uma das pesquisadoras, capacitada previamente, sob a supervisão de outra pesquisadora.

Os dados coletados foram anexados em planilha Microsoft Excel e distribuídos por mês e, posteriormente, foram analisados pelo programa de tratamento estatístico Statistical Package for the Social Sciences (SPSS). Para a análise estatística utilizou-se média e desvio padrão para realizar a análise descritiva dos dados, médias mensais e as médias anuais de 2015 e 2016. O teste T-Student foi empregado para comparar os dados referentes aos meses do estudo e um estudo de séries temporais para a análise das médias mensais.

O estudo atendeu à Resolução 466/2012 do Conselho Nacional de Saúde sobre pesquisa com seres humanos e teve a 
aprovação do Comitê de Ética e Pesquisa (CEP). Integra um projeto denominado "Gestão do cuidado de enfermagem em uma unidade neonatal na perspectiva da qualidade e segurança", aprovado pelo Comitê de Ética do hospital (campo de estudo) e cadastrado na Plataforma Brasil.

\section{Resultados}

Foram obtidas 15.012 medidas de escore do NAS, compreendidas do período de primeiro de janeiro de 2015 a 31 de dezembro de 2016. Em 2015 encontrou-se média de 596 medidas mensais e, no ano de 2016, média de 655 medidas mensais. A frequência absoluta mostrou um acréscimo de 712 registros no ano de 2016 em comparação ao ano de 2015 (Tabela 1).

Tabela 1 - Registros mensais do NAS nos anos 2015-2016, Porto Alegre, Brasil.

\begin{tabular}{lll}
\hline Mês & 2015 & 2016 \\
\hline Janeiro & 571 & 612 \\
Fevereiro & 587 & 616 \\
Março & 644 & 762 \\
Abril & 537 & 612 \\
Maio & 625 & 693 \\
Junho & 584 & 561 \\
Julho & 535 & 828 \\
Agosto & 603 & 647 \\
Setembro & 588 & 463 \\
Outubro & 543 & 709 \\
Novembro & 604 & 701 \\
Dezembro & 729 & 658 \\
\hline TOTAL & 7150 & 7862 \\
\hline
\end{tabular}

Fonte: Dados extraídos do sistema institucional do banco de dados do NAS.

A média mensal encontrada em 2015 corresponde a 19,86 registros diários de NAS. A rotina de avaliação do NAS prevê que seja realizada uma vez ao dia, sendo assim, diariamente foram avaliados cerca de 20 recém-nascidos. Ao utilizar o mesmo raciocínio para os dados de 2016, constata-se que ocorreram 21,83 avaliações diárias. Os resultados do ano de 2016 apontam que a unidade operou em capacidade elevada quanto à disponibilidade de leitos e de funcionalidade geral.

No que se refere a média de escore do NAS, em 2015, foi de 70,5, equivalente a 16,92 horas de assistência direta e indireta de enfermagem ao $\mathrm{RN}$ nas 24 horas do dia, e, no ano de 2016, a média foi de 78,8, traduzidos em 18,90 horas de assistência. A aplicação do teste T para amostras independentes constatou o coeficiente Pearson com uma diferença estatisticamente significativa, ou seja, $\mathrm{p}<0,001$ (Tabela 2). 
Tabela 2 - Médias de escore NAS mensal em 2015-2016, Porto Alegre, Brasil.

\begin{tabular}{lllll}
\hline \multirow{2}{*}{ Mês } & 2015 & & P \\
\cline { 2 - 3 } \cline { 5 - 5 } Janeiro & $73,1(14,4)$ & & $62,3(17,5)$ & $<0,001$ \\
Fevereiro & $71,8(13,8)$ & & $69,1(18,3)$ & 0,004 \\
Março & $69,9(10,9)$ & & $70,8(19,4)$ & 0,262 \\
Abril & $72,9(13,8)$ & & $69,2(16,5)$ & $<0,001$ \\
Maio & $73,3(14,5)$ & & $73,8(20,3)$ & 0,598 \\
Junho & $69,7(14,6)$ & & $81,7(19,6)$ & $<0,001$ \\
Julho & $69,0(17,3)$ & & $82,3(18,7)$ & $<0,001$ \\
Agosto & $71,6(17,5)$ & & $87,0(22,3)$ & $<0,001$ \\
Setembro & $74,8(13,6)$ & & $87,5(21,8)$ & $<0,001$ \\
Outubro & $70,9(12,5)$ & & $87,5(19,5)$ & $<0,001$ \\
Novembro & $70,2(17,5)$ & & $84,2(20,1)$ & $<0,001$ \\
Dezembro & $61,2(17,0)$ & & $85,0(19,6)$ & $<0,001$ \\
\hline Jun-Dez & $69,3(16,6)$ & $84,6(20,3)$ & $<0,001$ \\
\hline TOTAL & $70,5(15,4)$ & $78,8(21,1)$ & $<0,001$ \\
\hline
\end{tabular}

Fonte: Dados extraídos do sistema institucional do banco de dados do NAS.

Isolando o período posterior à capacitação (junho a dezembro de 2016) e os mesmos meses em 2015 e aplicando novamente o Teste $\mathrm{T}$ para amostras independentes também foi constatado um coeficiente Pearson com uma diferença estatisticamente significativa. Nesse segundo Teste T, os meses de janeiro, fevereiro, março, abril e maio de 2016 não foram incluídos, com o objetivo de não haver vieses no Teste $\mathrm{T}$ em decorrência de analisar períodos de sazonalidade distintos com relação à intervenção educativa. Ainda falando-se de valores dos escores de NAS, o gráfico do ano de 2016 (Gráfico 1) mostra que os valores dos escores do NAS estão mais uniformemente distribuídos entre 50 e 150 pontos quando comparados aos escores do ano de 2015, aos quais praticamente concentram entre 50 e 100 pontos na escala NAS.

Gráfico 1 - Comparativo dos escores NAS de junho a dezembro de 2015-2016, Porto Alegre, Brasil.

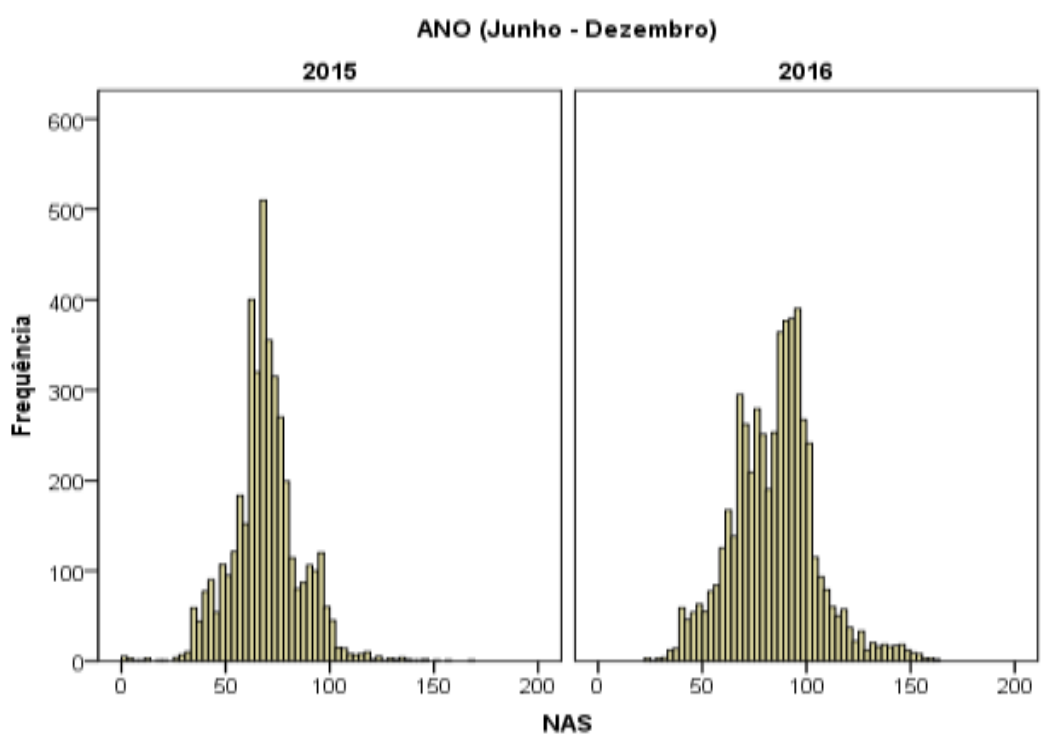

Fonte: Autores. 
Após o mês da capacitação, ou seja, maio, os escores de 2015 e 2016 foram se afastando em sentidos opostos, sendo 2016 se aproximando da média de escore preconizada pela Resolução COFEN n ${ }^{\circ}$ 0543/2017 que preconiza 18 horas de cuidados de enfermagem, por dia, para cuidados intensivos (Gráfico 2). Os valores de NAS, quando multiplicados por 14,4 min (14 min e $24 \mathrm{seg}$ ), permitem identificar o quantitativo de horas de cuidados de enfermagem demandados pelos pacientes por dia, isto é, os escores são convertidos em horas (Tabela 3). A média das horas de assistência em 2015 e 2016, quando isolado o período após a intervenção educativa, revela o valor de 20,30 horas, sendo que o mesmo período em 2015 obteve média de 16,63 horas. Assim, pode-se verificar que houve um acréscimo de 3,67 horas, no que se refere a horas de cuidados de enfermagem por RN.

Gráfico 2 - Comparação dos escores de NAS, 2015-2016, Porto Alegre, Brasil.

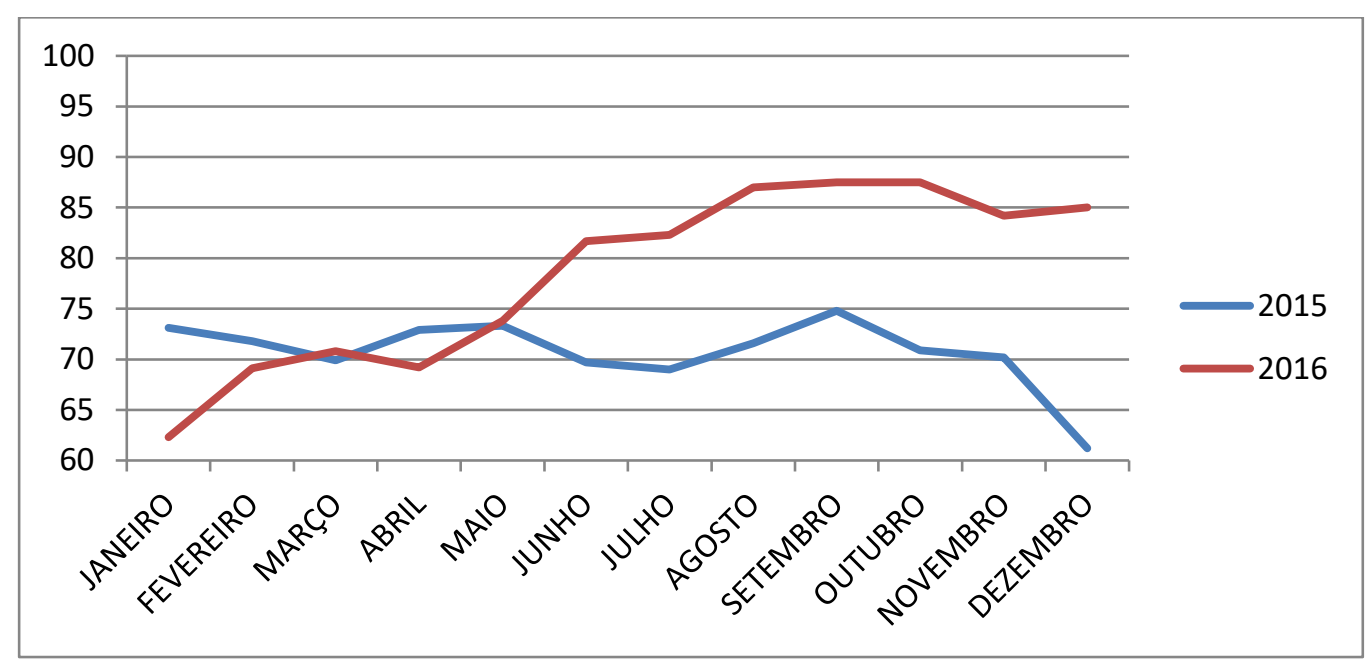

Fonte: Autores.

Tabela 3 - Média de horas de assistência por dia nos anos 2015-2016, Porto Alegre, Brasil.

\begin{tabular}{lllll}
\hline Mês & 2015 & 2015 & 2016 & 2016 \\
\cline { 2 - 2 } & horas & Média (h) & horas & Média (h) \\
\hline Janeiro & 17,56 & & 16,81 & \\
Fevereiro & 17,22 & & 16,88 & \\
Março & 16,77 & 17,33 & 17,15 & 17,08 \\
Abril & 17,51 & & 16,64 & \\
Maio & 17,6 & & 17,93 & \\
Junho & 16,72 & & 19,43 & \\
Julho & 16,41 & & 19,73 & \\
Agosto & 17,14 & & 21 & \\
Setembro & 18 & 16,63 & 21,16 & 20,3 \\
Outubro & 17 & & 21,16 & \\
Novembro & 16,85 & & 20,52 & \\
Dezembro & 15 & 20,37 & \\
\hline TOTAL & 16,98 & & 19,06 & \\
\hline \hline
\end{tabular}

Fonte: Dados extraídos do sistema institucional do banco de dados do NAS.

\section{Discussão}

O estudo aqui apresentado buscou comparar os escores de NAS nos anos de 2015 e 2016, considerando a ocorrência de uma intervenção educativa para uso do instrumento, realizada no mês de maio de 2016. Embora a mesma tenha sido desenvolvida 
respeitando o horário de trabalho dos profissionais, verificou-se que compareceram em torno de $72 \%$ das enfermeiras da unidade. A dificuldade em abranger a totalidade de enfermeiros em atividades educacionais tem sido relacionada a fatores como desmotivação e falta de interesse pessoal, assim como decorrente da pouca divulgação e da não liberação pela chefia para participar destas atividades (Macedo et al., 2019).

No caso específico do cenário aqui analisado, cabe esclarecer que os enfermeiros foram estimulados e liberados para participarem. Entretanto, acredita-se que abranger $100 \%$ dos profissionais de enfermagem nas atividades educativas ainda é um imenso desafio para os serviços de saúde, visto que o trabalho em turnos, a dupla jornada de trabalho, as escalas de férias e folgas dos setores e, ainda, as licenças para tratamento de saúde, quer sejam eventuais ou prolongadas, compõem uma equação de complexa resolução.

Os dados referentes ao NAS são de extrema importância para o gerenciamento do cuidado em enfermagem neonatal, visto que fundamentam o dimensionamento de pessoal da equipe de enfermagem e permitem o adequado planejamento da composição da equipe de enfermagem para atender as demandas de cuidado dos pequenos pacientes. A carga de trabalho média diária da unidade revelada, quando o escore do NAS é convertido em horas de assistência/paciente, pode ser aplicada nos modelos para o dimensionamento da equipe de enfermagem. A fórmula de Gaidzinsk, utilizada no contexto brasileiro há vários anos, considera as horas de cuidado demandadas pelos pacientes juntamente com outras variáveis e o resultado encontrado elucida o número de profissionais necessários para uma unidade realizar os cuidados diretos e indiretos adequados para cada paciente (Gaidzinski et al., 2009; Fugulin et al., 2016). O equilíbrio entre a demanda por cuidados e a força de trabalho de enfermagem disponível contribui para evitar sobrecarga de trabalho nos profissionais.

A inadequação numérica do pessoal de enfermagem, isto é, uma equipe subdimensionada, eleva a carga de trabalho por profissional, no entanto, dentre as possibilidades de se evitar danos na assistência, está a quantidade adequada de recursos humanos. O dimensionamento de pessoal objetiva, também, a garantir uma assistência de enfermagem de excelência e com menores índices de danos, assim como pode contribuir para a redução de riscos laborais (Branco et al., 2017).

Com relação a frequência de medições do NAS em 24 horas, pode-se observar nos resultados do presente estudo que em 2015 o setor trabalhou com taxa próxima a 100\% de ocupação e, em 2016, superou esse percentual. Neste sentido, os dados de 2016 correspondem a um acréscimo diário de, em média, duas avaliações de RN por dia na unidade em comparação com o ano de 2015. Na perspectiva da CTE, esse resultado revela dois RN em leitos extras na unidade demandando por cuidados de enfermagem intensivos. Além disto, há de ser reconhecido que as enfermeiras, ao avaliar diariamente os pacientes quanto às suas necessidades de cuidados de enfermagem por meio do NAS, estão cumprindo o artigo 49 (Seção IX) determinado pela Resolução $\mathrm{n}^{\mathrm{o}}$ 7, de 24 de fevereiro de 2010 (Brasil, 2010).

Por outro lado, os indicadores institucionais coletados no sistema informatizado registram uma taxa de ocupação funcional de 96,36\% e 96,58\% nos dois períodos anuais. A taxa de ocupação é um indicador utilizado no gerenciamento da área hospitalar, constituindo-se, tal como outros indicadores, em uma ferramenta para medir o desempenho e monitorar a performance dos serviços de saúde (Bao et al., 2019).

Acredita-se que a diferença encontrada entre a frequência de medidas de NAS (que apontam para uma ocupação de leitos em torno de $100 \%)$ e as taxas de ocupação funcional $(96,36 \%$ e $96,58 \%)$ possa estar relacionada ao gerenciamento dos leitos no setor ao longo do dia. Sabe-se que há uma variabilidade na ocupação dos leitos ao longo das 24 horas, visto que, muitas vezes o setor já está funcionando com sua capacidade máxima e ocorrem nascimentos na urgência ou partos gemelares, provocando na unidade uma superlotação transitória. Já os indicadores institucionais, incluindo a taxa de ocupação funcional, são calculados a partir do censo hospitalar realizado às 24 horas.

Ainda analisando as medições do NAS e a ocupação dos leitos à luz das orientações oficiais do Ministério da Saúde, a Portaria nº 1.020, de maio de 2013, que institui as diretrizes para a organização da Atenção à Saúde na Gestação de Alto Risco, 
estabelece que os serviços hospitalares para gestantes de alto risco devem manter uma taxa de ocupação mínima de $85 \%$ para os leitos obstétricos e 90\% para os leitos de UTIN, UCINCo e UCINCa (Brasil, 2013). Assim, apesar da taxa de ocupação da unidade estudada e as medições do NAS estarem em conformidade com a legislação vigente, há de ser ponderado que nos momentos em que o setor opera acima da capacidade instalada constituem-se em situações de vulnerabilidade para a ocorrência de erros assistenciais, comprometendo a segurança dos pacientes, pois as condições estruturais não acompanham as demandas assistenciais decorrentes de nascimentos imprevistos nas urgências obstétricas.

Os escores do NAS revelaram valores anuais com diferença, entre os períodos 2015 e 2016, estatisticamente significativa. Na prática, há o acréscimo de duas horas diárias por paciente, impactando na assistência direta e indireta de enfermagem. Observa-se que o mês de dezembro de 2015 foi o mês com menor escore entre os dois anos estudados, com escore NAS traduzindo-se em 15 horas de assistência de enfermagem por RN/dia, valor aquém do preconizado pela Resolução do COFEN 0543/2017, de 18 horas de enfermagem, por paciente, no cuidado intensivo (COFEN, 2017). Os meses de setembro e outubro de 2016 apresentaram os maiores escores do NAS (21,16 horas de assistência), apresentando valores que ultrapassaram o quantitativo preconizado pela Resolução COFEn 054/2017 e evidenciando maior carga de trabalho para a equipe de enfermagem nesse período.

A variabilidade nos resultados de NAS também tem instigado a realização de estudos. Em São Paulo, por exemplo, estudo em UTI de queimados identificou que a carga de trabalho de enfermagem foi influenciada por aspectos clínicos dos pacientes e desfechos da internação (Amadeu et al., 2020.) No sul do Brasil, pesquisa constatou valor médio de NAS de 70,0, com variações de 87,2 e 94,5 no primeiro e último dia de internação respectivamente (Freitas et al., 2020).

A intervenção educativa realizada em maio de 2016 teve por objetivo atualizar as enfermeiras da UTI Neonatal na aplicação do instrumento e, assim, uniformizar as avaliações realizadas pelas profissionais. A estratégia empregada, culminou com o aumento da pontuação, do NAS. A diferença entre os valores representa, aproximadamente, 4 horas adicionais de assistência entre os períodos analisados, valores com diferença estatística significativa e também prática, pois acrescem 4 horas ao cuidado para cada $\mathrm{RN}$.

Os escores de NAS encontrados após o mês da atividade educativa divergem de avaliações de RN em UTIN no interior de São Paulo, com valor médio de 62\% (14,8 horas) (Serafim et al., 2020). Outro estudo em UTIN, também brasileiro, encontrou valores médios de NAS que variaram entre $41,9 \%$ e 45,9\% (Soares et al., 2021). Quando se analisam estes escores, há de ser considerado possíveis diferenças nos perfis de gravidade e demandas por cuidados dos pequenos pacientes atendidos nestas instituições e níveis de UTI. Por outro lado, os valores pós intervenção aproximam-se daqueles em UTI de queimados com escores médios do NAS de 20,2 horas (Amadeu et al., 2020).

A atividade educativa impactou no processo de avaliação das enfermeiras frente ao RN, pois além de contribuir para homogeneizar o processo de atribuição de pontos, também provocou elevação dos resultados de NAS e, consequentemente, contribuiu para elucidar, de maneira mais fidedigna, a real carga de trabalho da unidade estudada. A presente investigação alinhase a outros estudos na área neonatal que reforçam a necessidade de um processo educativo para a utilização de instrumentos como o NAS (Mendes-Rodrigues et al., 2017). Assim, construiu-se numa estratégia para aprimoramento da assistência e melhoria da qualidade do atendimento oferecido aos pacientes (Silva et al., 2020).

Os dados obtidos no sistema informatizado da instituição não permitiram análises adicionais que possibilitassem explicar as diferenças encontradas nos meses de setembro e outubro (maiores escores) e dezembro (menores escores), o que foi considerado pelas pesquisadoras uma restrição no presente estudo. Outra limitação refere-se ao curto período de tempo póscapacitação (7 meses); porém, este foi um fator que não pode ser controlado pela equipe de pesquisa, pois havia uma delimitação de tempo para sua finalização. Sugere-se, assim, a continuidade da monitorização dos escores, visto que o grupo de enfermeiras pode ser modificado em virtude do desligamento de profissionais e com o ingresso de novas enfermeiras. E, além disto, 
considerando o tempo decorrido, de que possa haver efeitos tardios daquela intervenção.

O presente estudo contribui para a área de enfermagem na medida em que apresenta uma variação estatisticamente significativa dos escores do NAS após uma intervenção educativa realizada com enfermeiras. Esse fato reforça que a educação continuada ocupa um papel relevante na qualidade dos registros e dos cuidados realizados pelos enfermeiros, e que mesmo em situações onde os enfermeiros possuem condições mínimas de trabalho em instituições estruturadas, o fornecimento e revisão do conhecimento sobre as ferramentas utilizadas no cotidiano permanentemente se faz necessário.

No que tange à saúde, principalmente dos RN, os resultados desse estudo sugerem que haviam valores subestimados de carga de trabalho da equipe de enfermagem, podendo impactar negativamente na gestão dos recursos humanos de enfermagem e na segurança dos cuidados prestados. Dessa maneira, ao demonstrar que uma intervenção educativa pode impactar na qualidade da informação e no apoio a tomada de decisão, o presente estudo traz aspectos poucos explorados na aplicação das escalas, que concerne não só na importância da utilização dessas ferramentas, mas também na relevância da qualidade na aplicação dos instrumentos.

\section{Conclusão}

Através deste estudo, pode-se concluir que a intervenção educativa impactou na elevação dos escores anuais de NAS, aproximando as horas de enfermagem evienciadas por meio deste instrumento (19,06 horas por RN/dia) àquelas preconizadas pelo COFEn. Os resultados foram ainda mais impactantes quando foi isolado o período relativo aos meses pós-intervenção, revelando 20,03 horas por RN/dia. Assim, tanto da perspectiva de análise da média anual, quanto da análise oriunda das médias mensais, quando isolado o período pós-intervenção, evidencia-se o impacto positivo advindo da atividade de educação com as enfermeiras, ratificando a necessidade da educação permanente para os profissionais nos serviços de saúde.

O uso do NAS em UTI é de extrema importância, pois um dimensionamento adequado favorece a assistência integral ao $\mathrm{RN}$ e aos seus familiares, que demandam por cuidados que vão além do atendimento às necessidades físicas, requerendo a adoção de comportamentos dos profissionais que contemplem práticas de humanização inseridas no ambiente da UTIN. Em relação à equipe de enfermagem, um dimensionamento adequado evita sobrecarga de trabalho para estes profissionais que, por sua vez, pode levar ao adoecimento do profissional.

Sugere-se, como proposição para futuro estudo, a análise acerca da necessidade da elucidação dos itens do instrumento que possuem pontuação mais elevada, ou seja, em quais processos diretos e/ou indiretos há maior carga de trabalho. Assim, além do instrumento subsidiar um dimensionamento de pessoal adequado à realidade da unidade, também poderá auxiliar num melhor ajuste da alocação dos profissionais para atividades as quais demandem maior tempo de enfermagem.

\section{Agradecimentos}

O presente estudo recebeu fomento do Fundo de Incentivo à Pesquisa do Hospital de Clínicas de Porto Alegre. A equipe de investigação agradece as enfermeiras da Unidade de Neonatologia do Hospital de Clínicas de Porto Alegre pela sua participação nas atividades de capacitação, pelo interesse demonstrado em melhorar a assistência prestada aos recém-nascidos e por contribuírem com a construção do conhecimento em enfermagem. Procede-se também o agradecimento ao Grupo de Pesquisa e Pós-Graduação do Hospital de Clínicas de Porto Alegre pelo apoio na realização do estudo.

\section{Referências}

Amadeu, L. M., Dell'Acqua, M. C. Q., Castro, M. C. N., Palhares, V. C., Serafim, C. T. R., \& Trettene, A. S. (2020). Nursing workload in burn intensive care unit. Rev Bras Enferm, 73(Suppl 1), e20190446. 
Aragão, N. S. C., Barbosa, G. B. B., Santos, C. L. C., Nascimento, D. S. S., Vilas Bôas, L. B. S., Martins, D. F., et al. (2021). Burnout Syndrome and Associated Factors in Intensive Care Unit Nurses. Rev Bras Enferm, 74 (Suppl 3), e20190535.

Báo, A. C. P., Amestoy, S. C., Moura, G. M. S. S., \& Trindade, L. L. (2019). Indicadores de qualidade: ferramentas para o gerenciamento de boas práticas em saúde. Rev Bras Enferm, 72 (2).

Biff, D., Pires, D. E. P., Forte, E. C. N., Trindade, L. L., Machado, R. R., Amadigi, F. R., et al. (2020). Cargas de trabalho de enfermeiros: luzes e sombras na Estratégia Saúde da Família. Ciência \& Saúde Coletiva, 25 (1), 147-158.

Bochembuzio, L. (2007). Avaliação do instrumento Nursing Activities Score (NAS) em neonatologia. Tese de Doutorado, Escola de Enfermagem, Universidade de São Paulo, São Paulo.

Branco, L. L. W. V., Beleza, L. O. \& Luna, A. A. (2017). Carga de trabalho de enfermagem em UTI neonatal: aplicação da ferramenta. Rev Fund Care Online, $9(1), 144-151$.

Brasil. (2010). Ministério da Saúde. Resolução-RDC no 7, 24 de fevereiro de 2010. 2010 fev,37. Seção 1. p 48.

Brasil. (2013). Ministério Da Saúde. Portaria Nº 1.020, 29 de maio de 2013. Brasília, V. Institui as diretrizes para a organização da Atenção à Saúde na Gestação de Alto Risco e define os critérios para a implantação e habilitação dos serviços de referência à Atenção à Saúde na Gestação de Alto Risco, incluída a Casa de Gestante, Bebê e Puérpera (CGBP), em conformidade com a Rede Cegonha. 2013 maio. Seção 2.

Bruyneel, A., Tack, J., Droguet, M., Maes, J., Wittebole, X., Miranda, R., Di Pierdomenico, L. (2019). Measuring the nursing workload in intensive care with the Nursing Activities Score (NAS): A prospective study in 16 hospitals in Belgium. Journal of Critical Care, 54, $205-211$.

Castro, M. C. N., Dell'Acqua, M. C. Q., Unger, I. C., Cyrino, C. M. S., \& Almeida, P. M. V. (2018). Gravidade e carga de trabalho de enfermagem. Esc Anna Nery, 22 (1), e20170167.

Conselho Federal de Enfermagem. Resolução COFEN n ${ }^{\circ}$ 543/2017, de 18 de abril de 2017. Atualiza e estabelece parâmetros para o Dimensionamento do Quadro de Profissionais de Enfermagem nos serviços/locais em que são realizadas atividades de enfermagem. Brasília: COFEN, 2017.

Freitas, E. de O., Magnago, T. S. B de S., Magalhães, A. M. M de, Andolhe, R., Cunha, Q. B. da, \& Camponogara, S. (2020). Carga de trabalho da enfermagem em unidade de terapia intensiva: um estudo de método misto. Research, Society and Development, 9 (1), e161911733.

Fugulin, F. M. T., Gaidzinski, R. R. \& Lima, A. F. C. (2016). Dimensionamento de pessoal de enfermagem em instituições de saúde. In: Kurcgant, Paulina. Gerenciamento em enfermagem. Rio de Janeiro: Guanabara Koogan.

Gaidzinski, R. R., Fugulin, F. M. T., Peres, H. H. C., Castilho, V., Massarolo, M. C. K. B., Mira, V. L., Pereira, I. M., \& Tsukamoto, R. (2009). Dimensionamento informatizado de profissionais de enfermagem: inovação tecnológica. Rev Esc Enferm USP, 43 (2), 1314-9.

Guzzo, G. M., Magalhães, A. M. M., Moura, G. M. S. S., \& Wegner, W. (2018). Segurança da terapia medicamentosa em neonatologia: olhar da enfermagem na perspectiva do pensamento ecológico restaurativo. Texto e Contexto Enferm, 27 (3), e4500016.

Hoffmeister, L. V., Moura, G. M. S. S. \& Macedo, A. P. M. C. (2019). Learning from mistakes: analyzing incidents in a neonatal care unit. Rev. Latino-Am. Enfermagem, 27, e3121.

Kleba, M. E., Hoefle, N., Oliveira, G. M., \& Rodrigues, O. C. C. (2017). Strengthening the leadership of the Commission on Education-Service Integration for permanent health education. Rev Gaúcha Enferm, 38 (4), e2016-0008.

Macêdo, W. T. P., Figueiredo, B. M., Reis, D. S. T., Barros, S. H. P., Ramos, M. C. A., \& Silva, S. E. D. (2019). Adesão dos profissionais de enfermagem às práticas educacionais. Rev Fun Care Online, 11 (4), 1058-1064.

Magalhães, A. M. M., Costa, D. G., Riboldi, C. O., Mergen, T., Barbosa, A. S., \& Moura, G. M. S. S. (2017). Association between workload of the nursing staff and patient safety outcomes. Rev Esc Enferm USP, 51, e03255.

Mendes-Rodrigues, C., Costa, K. E. S., Antunes, A. V., Gomes, F. A., Rezende, G., \& Silva, D. V. (2017). Carga de trabalho e dimensionamento de pessoal de enfermagem em unidades de terapia intensiva. Rev. Aten. Saúde, 15 (53), 5-13.

Oliveira, P. M. V., NakahataK, S., \& MarquesI, R. (2019). Avaliação da demanda de intervenções de enfermagem em terapia intensiva segundo o Nursing Activities Score (NAS). Revista Eletrônica Acervo Saúde, 11 (10), e453.

Oliveira, E. M., Secco, L. M. D., Figueiredo, W. B., Padilha, K. G., \& Secoli, S. R. (2019). Nursing Activities Score and the cost of nursing care required and available. Rev Bras Enferm [Internet], 72 (Suppl 1), 137-42.

Queijo, A. F. \& Padilha, K. G. (2009). Nursing Activities Scores (NAS): adaptação transcultural e validação para a língua portuguesa. Rev. da Esc. de Enferm. da USP, 43 (especial), 18-25.

Santos, A. P. A., Carvalho, T., Soares, J. P. R., Coelho, P. R. L. F., \& Santos, E. S. (2021). Comparação da Carga de Trabalho de Enfermagem entre Pacientes Clínicos e Cirúrgicos em Terapia Intensiva. Cogitare enferm, v26, e73689.

Serafim, C. T. R., Rodrigues, G. M., Silveira, R. R. P., Castro, M. C. N., Dell'Acqua, M. G. Q., \& Lima, S. A. M. (2020). Nursing activities score como instrumento gerencial em unidade de terapia intensiva neonatal. Revista Norte Mineira de Enfermagem, 9 (2), 90-97.

Silva, A. P. B., Prado, M. A., Silva, L. C. S., Andrade, L. Z., Pascoa, H., Bastos, L. R. R., et al. (2021). O espaço de trabalho da enfermagem em Unidades Neonatal e os riscos laborais: revisão integrativa. Research, Society and Development, 10 (13), e566101321701.

Silva, C. P. G. da, Aperibense, P. G. G de S., Almeida Filho, A. J. de, Santos, T. C. F., Nelson, S., \& Peres, M. A. de A. (2020). Da educação em serviço à educação continuada em um hospital federal. Esc Anna Nery, 24 (4). 
Research, Society and Development, v. 10, n. 17, e126101723158, 2021

(CC BY 4.0) | ISSN 2525-3409 | DOI: http://dx.doi.org/10.33448/rsd-v10i17.23158

Silva, L. H. F., Santo, F. H. E., Chibante, C. L. P., \& Paiva, E. D. (2018). Permanent Education in a neonatal unit from Culture Circles. Rev Bras Enferm, 71 (Suppl 3), 1328-33.

Soares, M. K., Dorigan, G. H., \& Carmona, E. V. (2021). Carga de trabalho e dimensionamento em unidade de internação neonatal: uso do Nursing Activities Score. Enferm Foco, 12 (2), 250-5.

Tomazoni, A., Rocha, P. K., Ribeiro, M. B., Serapião, L. S., Souza, S. \& Manzo, B. F. (2017). Segurança do paciente na percepção da enfermagem e medicina em unidades de terapia intensiva neonatal. Rev Gaúcha Enferm, 38 (1), e64996. 